\title{
Korean Wide Area Differential Global Positioning System Development Status and Preliminary Test Results
}

\author{
Ho Yun* and Changdon Kee \\ School of Mechanical and Aerospace Engineering and the Institute of Advanced Aerospace Technology, Seoul National \\ University, Seoul 151-744, Korea
}

\section{Doyoon Kim}

Defense Acquisition Program Administration, Seoul, 151-744, Korea

\begin{abstract}
This paper is focused on dynamic modeling and control system design as well as vision based collision avoidance for multi-rotor unmanned aerial vehicles (UAVs). Multi-rotor UAVs are defined as rotary-winged UAVs with multiple rotors. These multi-rotor UAVs can be utilized in various military situations such as surveillance and reconnaissance. They can also be used for obtaining visual information from steep terrains or disaster sites. In this paper, a quad-rotor model is introduced as well as its control system, which is designed based on a proportional-integral-derivative controller and vision-based collision avoidance control system. Additionally, in order for a UAV to navigate safely in areas such as buildings and offices with a number of obstacles, there must be a collision avoidance algorithm installed in the UAV's hardware, which should include the detection of obstacles, avoidance maneuvering, etc. In this paper, the optical flow method, one of the vision-based collision avoidance techniques, is introduced, and multi-rotor UAV's collision avoidance simulations are described in various virtual environments in order to demonstrate its avoidance performance.
\end{abstract}

Key words: Wide area differential global positioning system, Differential global positioning system, Differential global navigation satellite system

\section{Introduction}

The satellite based augmentation system (SBAS) is one of the most widely used global navigation satellite system (GNSS) augmentation systems as Fig. 1 shows. The US began to develop wide area augmentation system (WAAS) in 1994, and recently they are trying to extend its coverage to South America. The European Space Agency, European Commission and EUROCONTROL have developed EGNOS, and the official start of the operation was announced on 1 October 2009. Japan also developed its own SBAS named multi-functional satellite augmentation system (MSAS) and began its full-operation. Many other countries including Korea are developing their own SBAS.
In Fig. 1, the country in the red circle is Korea. Because Korea is located between Japan and China, MSAS signals are available in Korea even though it is not officially certified

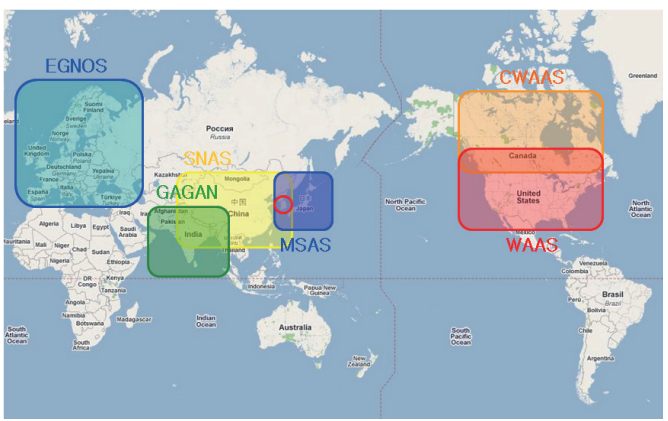

Fig. 1. Satellite based augmentation system status.

(c) * Corresponding author

E-mail: yunho3@snu.ac.kr Tel: +82-2-880-7395 Fax: +82-2-878-0559 
for Korean users. If China starts the satellite navigation augmentation system service in the future, it can also be used in Korea.

Figures 2 and 3 are the horizontal and vertical positioning results at Daejon, Korea. Daejon is located at the center of Korea. As Fig. 2 shows, both horizontal and vertical position accuracies are considerably improved. Therefore, it could be argued that it is not necessary for Korea to develop its own SBAS. However, MSAS performance is not certified in the Korean region, so using MSAS in Korea can cause a critical safety problem. Figure 4 is a triangular plot of horizontal/ vertical protection levels and position errors at Usuda in Japan and Fig. 5 is the same plot at Daejon in Korea.

Vertical/horizontal position errors are 1.19/1.99 meters in Japan. As mentioned above, in Korea, positioning performances are as good as in Japan (1.04/2.09 meters each). However, the protection level in Korea has a much higher value than in Japan, so that the availability of MSAS is very low in the Korean region. Localizer performance vertical (LPV) availability was $90.6 \%$ at Usuda, Japan, and $49.4 \%$ at

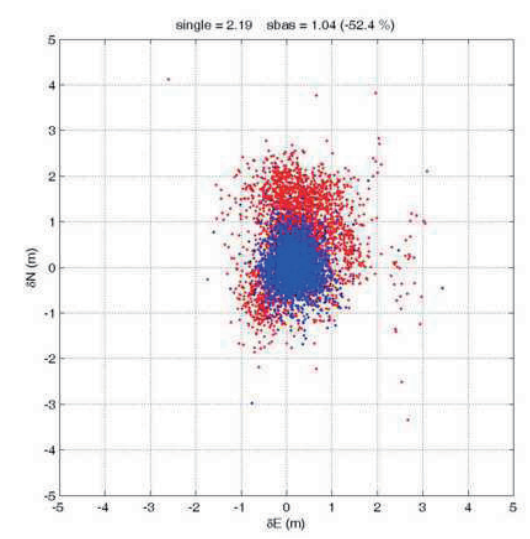

Fig. 2. Horizontal positioning result at daejon (red: standalone, blue: multi-functional satellite augmentation system).

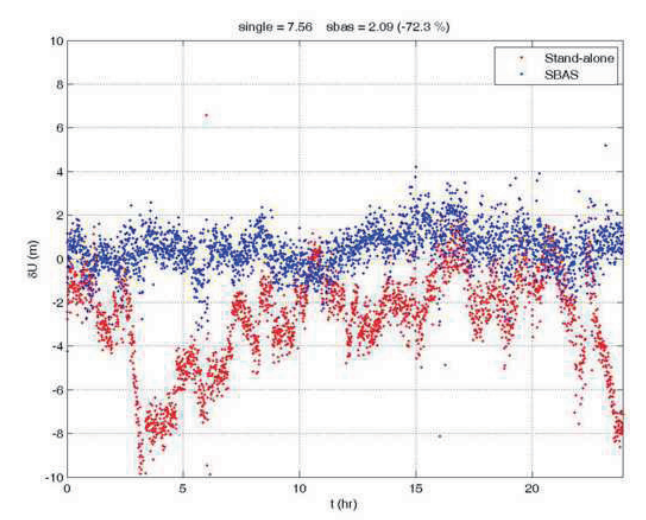

Fig. 3. Vertical positioning result at Daejon (red: standalone, blue: multi-functional satellite augmentation system).
Daejon in Korea. This is because Korea is located far from the center of the MSAS service area.

For this reason, the Korean government decided to develop its own system and recently resumed the wide area differential global positioning system (WADGPS) development project. The main goal of this project is to show the capability of Korean WADGPS using the pseudolite and existing NDGPS infrastructures in real-time. This project is scheduled for 2010 to 2014 under contract with the Ministry of Land, Transport and Maritime Affairs (MLTM). The pseudolite-based WDGPS demo system includes four wide-area reference stations (WRS) located in the NDGPS stations, one wide-area master station (WMS) in Seoul National University (SNU), and one pseudolite which will be broadcasting the augmenting message. Each element is described in the following section as well as current status and schedule of the project. Finally, preliminary test result will be described.

\section{Korean WADGPS Development Status}

\subsection{Past works}

Since 1999, the Korean MLTM has installed the NDGPS reference stations and has been providing local area DGPS service. Eleven coast reference stations and sux inland reference stations cover the whole area of South Korea. Nine monitoring stations are monitoring the status of DGPS
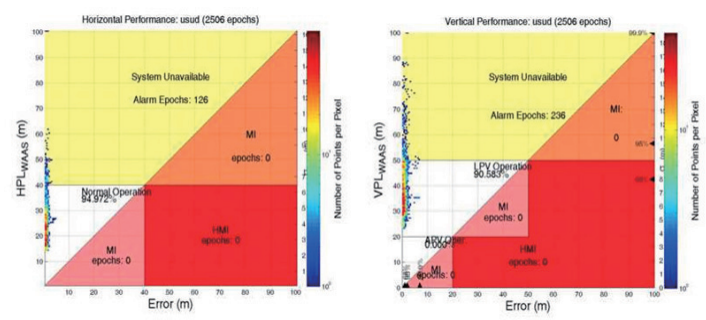

Fig. 4. Multi-functional satellite augmentation system performance in Japan (left: horizontal, right: vertical).
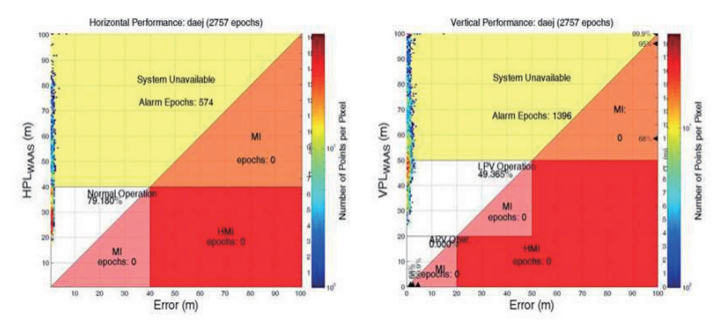

Fig. 5. Multi-functional satellite augmentation system performance in Korea (left: horizontal, right: vertical). 


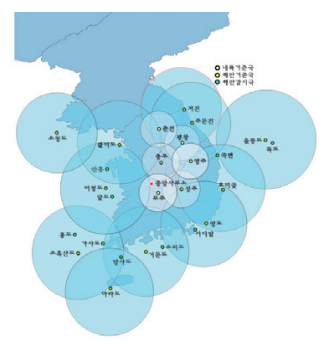

Fig. 6. National differential global positioning system reference stations in Korea.

Table 1. Current NDGPS NTRIP service status

\begin{tabular}{|c|c|c|c|}
\hline No & Mountpoint & Format & Nav_system \\
\hline 1 & DAEJ_RTCM20 & RTCM2.0 & DGPS \\
\hline 2 & DAEJ_RTCM23 & RTCM2.3 & DGPS+RTK \\
\hline 3 & YNJU_RTCM20 & RTCM2.0 & DGPS \\
\hline 4 & YNJU_RTCM23 & RTCM2.3 & DGPS+RTK \\
\hline 5 & MUJU_RTCM20 & RTCM2.0 & DGPS \\
\hline 6 & MOOJ_RTCM23 & RTCM2.3 & DGPS+RTK \\
\hline 7 & DOKD_RTCM23 & RTCM2.3 & DGPS+RTK \\
\hline 8 & SOCH_RTCM2O & RTCM2.0 & DGPS \\
\hline 9 & JUMN_RTCM20 & RTCM2.0 & DGPS \\
\hline 10 & JEOJ_RTCM20 & RTCM2.0 & DGPS \\
\hline 11 & SOHE_RTCM2O & RTCM2.0 & DGPS \\
\hline 12 & MARA_RTCM20 & RTCM2.0 & DGPS \\
\hline 13 & GEOM_RTCM20 & RTCM2.0 & DGPS \\
\hline 14 & YNDO_RTCM20 & RTCM2.0 & DGPS \\
\hline 15 & HOMI_RTCM2O & RTCM2.0 & DGPS \\
\hline 16 & CHUN_RTCM2O & RTCM2.0 & DGPS \\
\hline 17 & PYCH_RTCM20 & RTCM2.0 & DGPS \\
\hline 18 & CCHJ_RTCM23 & RTCM2.3 & DGPS+RTK \\
\hline 19 & CHUN_RTCM23 & RTCM2.3 & DGPS+RTK \\
\hline 20 & EOCH_RTCM2O & RTCM2.0 & DGPS \\
\hline 21 & CCHJ_RTCM23 & RTCM2.3 & DGPS+RTK \\
\hline 22 & PALM_RTCM20 & RTCM2.0 & DGPS \\
\hline 23 & PALM_RTCM23 & RTCM2.3 & DGPS+RTK \\
\hline 24 & DOKD_RTCM20 & RTCM2.0 & DGPS \\
\hline 25 & JEOJ_RTCM23 & RTCM2.3 & DGPS+RTK \\
\hline 26 & ULLE_RTCM20 & RTCM2.0 & DGPS \\
\hline
\end{tabular}

NDGPS: national differential global positioning system, NTRIP: networked transport of RTCM via internet protocol.

correction messages, so that DGPS users can receive safety certified service.

For the user who does not have a DGPS beacon receiver, each station also provides the correction messages in TCP/ IP protocol using networked transport of RTCM via internet protocol (NTRIP). Table 1 shows the status of NTRIP service by Korean NDGPS reference stations.

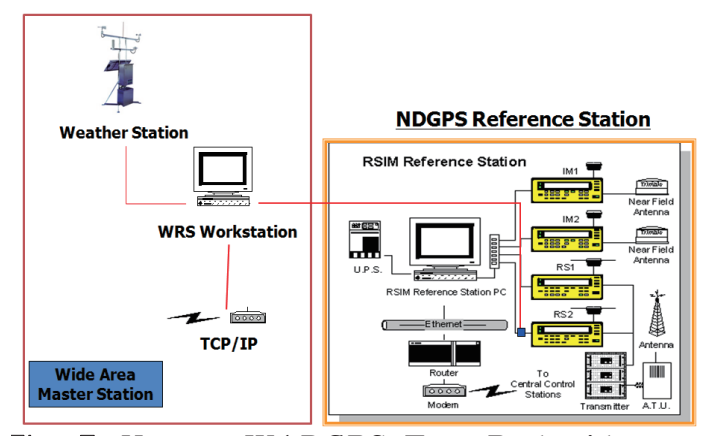

Fig. 7. Korean WADGPS Test Bed wide-area reference stations architecture. WADGPS: wide area differential global positioning system.

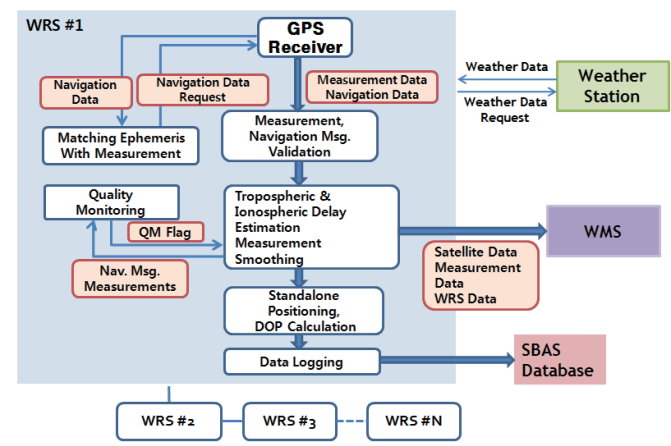

Fig. 8. Functionality wide-area reference station.

From 2003 to 2005, WADGPS research groups in SNU have developed the Korean WADGPS Test Bed (KWTB). The objectives of the KWTB are to develop the related essential technology, to verify the feasibility of Korean WADGPS. The test bed consists of four WRSs and one WMS. WRSs have been installed in the existing facilities of NDGPS reference stations.

As shown in Fig. 7, a WRS workstation has been installed with an NDGPS backup receiver so that test bed equipment has no interference on current NDGPS operations. WMS is an independent central processing element which has no attached receiver and gathers all the necessary data from WRSs via internet connection. Therefore, WMS have been installed in the SNU GNSS Lab because WMS needs no NDGPS infrastructure.

\subsection{Current status}

Figure 8 is a block diagram of WRS. WRS receives the measurements and navigation messages from the GPS receiver, and validates these data by quality monitoring.

Using these valid measurements, navigation data and weather data from weather stations, WRS calculates the 
basic information and transfers it to WMS. WRS also plays an added role as a permanent test user for monitoring and analyzing performance of the demo system including accuracy, integrity, availability, and continuity.

WMS receives the raw data and WRS data from the multiple WRSs. After time synchronization of multiple WRS data, it checks the integrity flags and determines the optimal issue of data ephemerides (IODE). Using raw data and WRS data from WRSs, WMS estimate the ionospheric grid point vertical delays (Chao, 1997). As in Eq. 1, the ionospheric delay which is estimated from reference station contains receiver interfrequency bias (Rx IFB) and transmitter IFB (Tx IFB).

$$
i_{i, s}^{j}=\bar{i}_{i, s}^{j}+\left(i_{b_{R x}, i}-i_{b_{T x}}^{j}\right)=\bar{i}_{i, v}^{j} Q_{i}^{j}+\left(i_{b_{R x}, i}-i_{b_{T x}}^{j}\right)
$$

Subscript i means the $i$-th reference station and superscript j means the $\mathrm{j}$-th satellite.

For implementing grid algorithms, both Rx IFB and Tx IFB should be estimated. Tx IFB can be easily eliminated using time of group delay value, which is from GPS navigation data. Eq. 2 is an Tx IFB compensated ionospheric delay.

$$
\tilde{i}_{i, s}^{j}=\bar{i}_{i, S}^{j}+i_{b_{R x}, i}=i_{i, v}^{j} Q_{i}^{j}+i_{b_{R x}, i}
$$

Fitting the ionosphere as spherical harmonics model, WMS estimates the Rx IFB of WRS. Eq. 2 shows the second order spherical harmonics model.

$$
\begin{gathered}
i_{y}\left(\phi_{M A}, \lambda\right)=\sum_{n=0}^{2}\left[\sum_{m=0}^{n}\left\{c_{m m} \cos m \lambda+S_{n m} \sin m \lambda\right\} P_{i n m}\left(\sin \phi_{M}\right)\right] \\
\operatorname{Pnn}(x)=\frac{1}{2^{n} \cdot n !}\left(1-x^{2}\right)^{2} \frac{d^{m}}{d x^{m}}\left[\frac{d^{n}}{d x^{n}}\left(x^{2}-1\right)^{n}\right]
\end{gathered}
$$

Substituting Eq. 2 for Eq. 3, ionospheric delay and Rx IFB can be modeled as a function of local time and geomagnetic latitude. For estimating the Rx IFB in real time, Kalman filter has been implemented. Eq. 4 has been used as an observation equation.

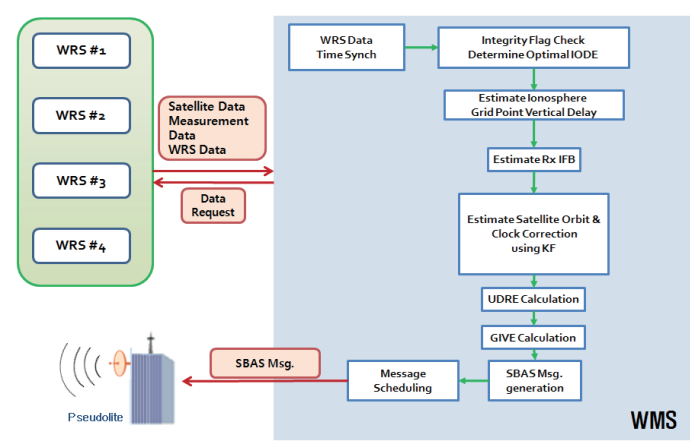

Fig. 9. Functionality wide-area reference station.

$$
\tilde{i}_{i, s}^{j}=\sum_{n=0}^{2}\left[\sum_{m=0}^{n}\left\{C_{n m} \cos m \lambda+S_{n m} \sin m \lambda\right\} P_{n m}\left(\sin \phi_{M L}\right)\right] Q_{i}^{j}+i_{b_{R,},}
$$

Satellite orbit and clock errors are estimated using inverted GPS methods with Kalman filter (Tsai, 1999). After estimating the correction it generates the integrity information and SBAS messages (Radio Technical Commission for Aeronautics, 2006; Walter et al., 2001).

For compatibility, each element of the system uses the NTRIP for exchanging necessary data.

From 2004 to 2005 a WADGPS post process was conducted using KWTB for verifying the feasibility. Four WRSs gathered and logged data from GPS satellites and weather stations. WMS used these data from WRSs, estimated the correction and the integrity information, and logged these augmentation messages. Users received an augmentation message from WMS, and used it when estimating their position. Figure 10 shows the results.

Hazardously misleading information rate was $0 \%$ and LPV availability was $99.87 \%$. This satisfies the WAAS initial operational capability requirement. Contrary to MSAS, KWTB has been developed for Korean users, therefore post processing results show that it has higher levels of performance in Korea, especially in availability aspects.

\subsection{Korean WADGPS development plan}

The Korean WADGPS development project was stopped in 2005 for various reasons. Although the project was stopped, many research institutes and universities kept developing the WADGPS algorithms and technologies and recently the project was resumed. The main goal of this project is to show the capability of Korean WADGPS using pseudolite and existing NDGPS infrastructures in real-time. This project is scheduled for 2010 to 2014 under the contract with the MLTM. The Korean government developed the National GNSS Master Plan and Space Vision 2016 program to support and coordinate the GNSS development in Korea. According to this plan, after this project, Korea will launch
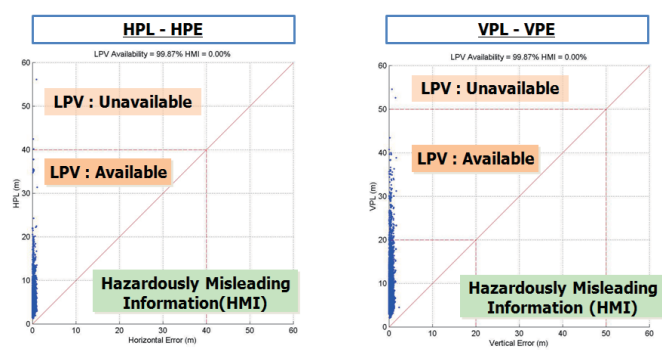

Fig. 10. User performance with Korean WADGPS Test Bed. WADGPS: wide area differential global positioning system. 
a geostationary multifunctional satellite with a navigation payload which will be broadcasting augmenting signals. This project is under active development to satisfy the following objectives:

- Increased overall navigation performance (land/ air/ marine, civil/military)

- Independent \& interoperable with other SBASs

- Certified quality of service

- Qualified for safety critical applications

MLTM and Korea Institute of Marine Science \& Technology (KIMST) promotion provide funding and manage the project. SNU is developing the WMS main system, and Korea Aerospace University (KAU) is improving the ionosphere

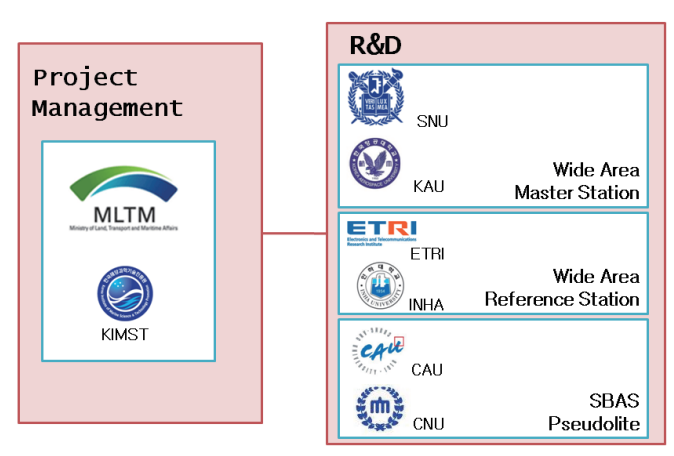

Fig. 11. Korean wide area differential global positioning system development project framework. and troposphere related algorithms. The Electronics and Telecommunications Research Institute (ETRI) takes WRS main system development, and Inha University is developing the WRS surroundings and circumstance monitoring system. The Pseudolite broadcasting system will be developed by Chung-Ang University (CAU) with the help of Chungnam National University (CNU).

Figure 12 describes pseudolite-based WADGPS demo system architecture, which is being developed now. Performances of WRS and WMS will be improved during this project. In KWTB, some integrity function is not implemented yet, and there is much room for improvement. Automated WRS surroundings and circumstance monitoring system will be developed and WRS integrity related module will be improved. WAAS message type 10, 28 will be adopted in

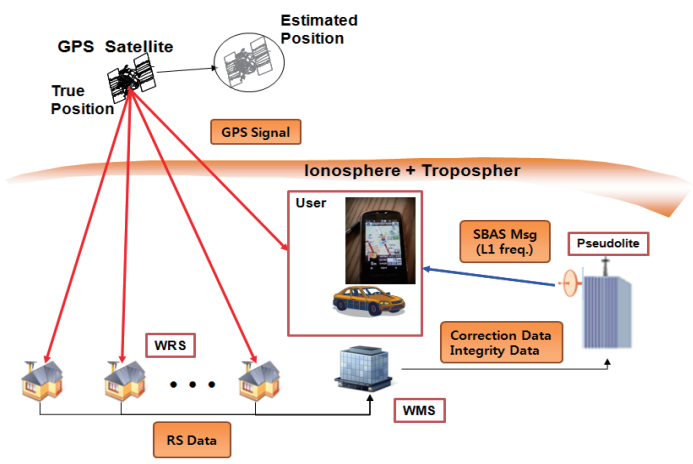

Fig. 12. Pseudolite-based wide area differential global positioning system demo system architecture.

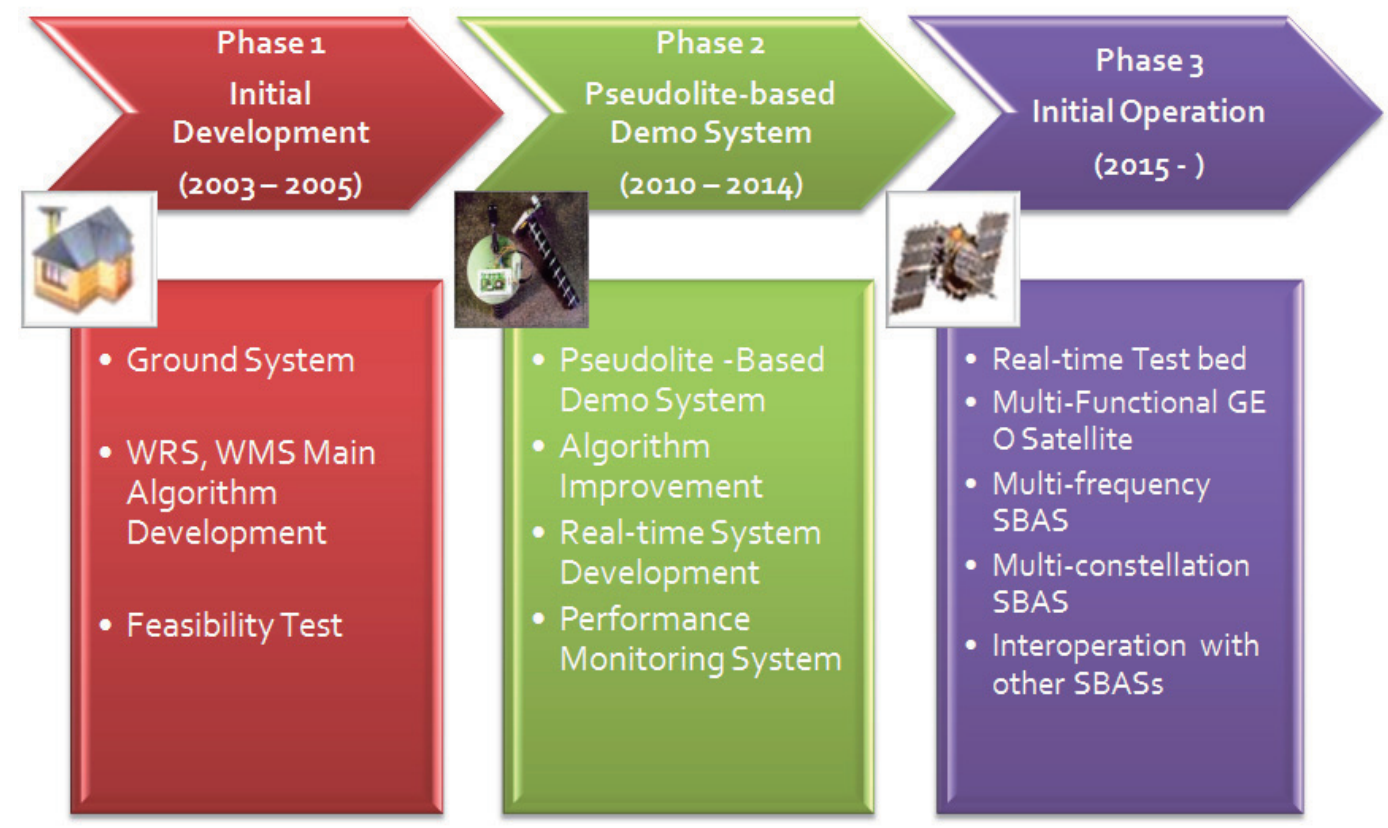

Fig. 13. Milestones of Korean wide area differential global positioning system development. 


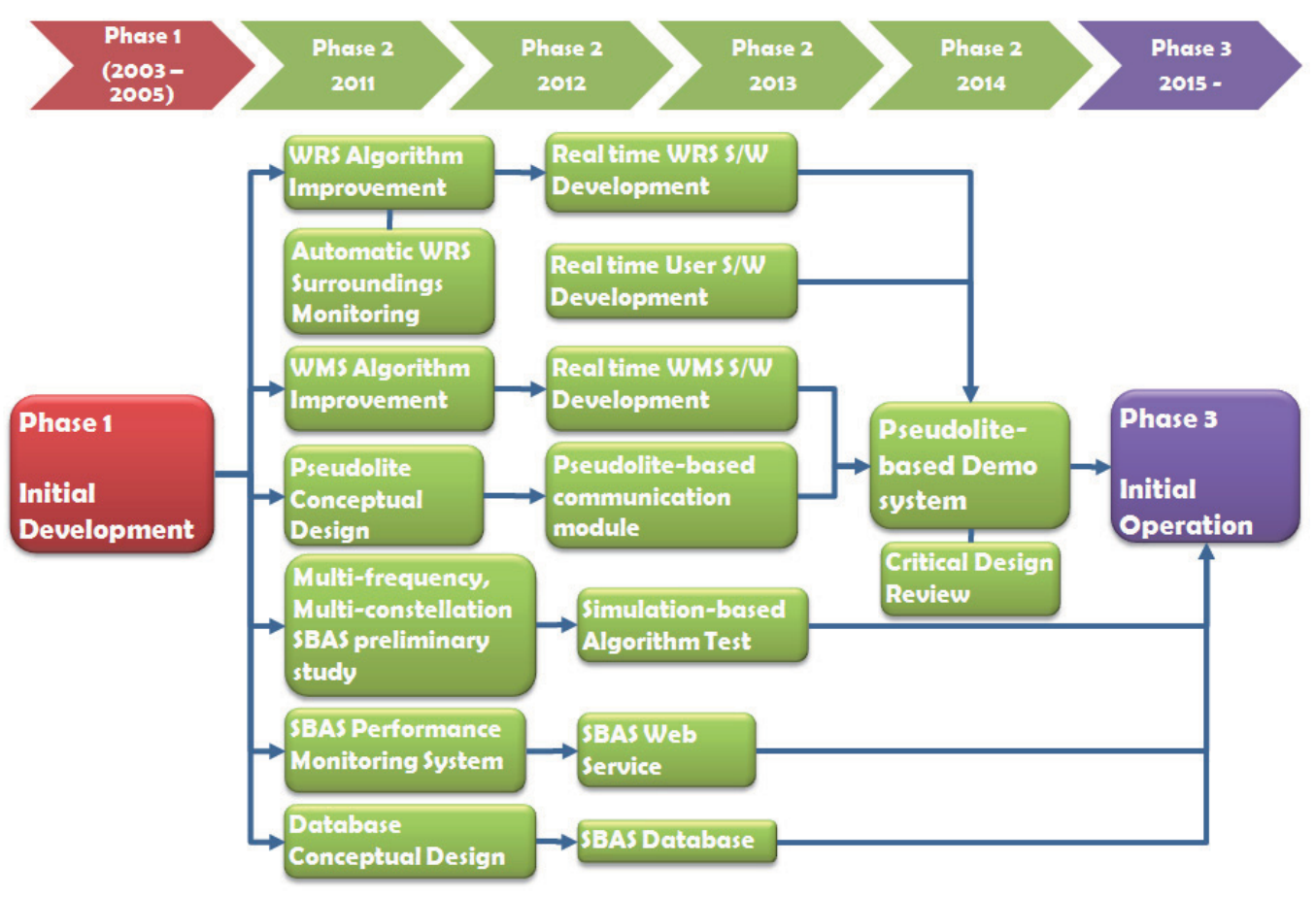

Fig. 14. Project schedule (phase 2).

KWTB WMS integrity module, according to WAAS minimum operational performance standards(MOPS) and other related standards (Radio Technical Commission for Aeronautics, 2006; Walter et al., 2001). Pseudolite receives the augmenting message from WMS, modulates it to L1 frequency and broadcasts it to users so that it can play the same role as geosynchronous orbit (GEO) satellite. By broadcasting the augmenting message using a pseudolite signal, experiments can be conducted in almost the same environment as a real SBAS. Finally, user tests will be undertaken with a commercial smart phone to show the compatibility of the system.

After this project, a GEO satellite will be launched, and the pseudolite broadcasting system can be used in the location where the GEO satellite signals are blocked because of geographical features or obstacles. This pseudolite broadcasting system can solve the SBAS GEO satellite visibility problem effectively.

As Fig. 13 shows, The Korean WADGPS development project can be divided into 3 phases. Phase 1 is the initial development phase. In this phase we develop the WRS, WMS main algorithms and secured the core technologies of ground systems successfully.

Currently, the project is in phase 2 , in which some algorithms are improved. In this phase the real-time system and performance monitoring system will be developed.
Finally, the technology of the ground system and pseudolite broadcasting system are secured in phase 2 . In addition, the preliminary study of multi-frequency/multi-constellation SBAS will be conducted for the future. Figure 14 shows a detailed master plan of phase 2 .

After phase 2, a multi-functional GEO satellite will be launched according to the National GNSS Master Plan and Space Vision 2016 program. In this phase, Korean SBAS will start the initial operation and the algorithms will be developed to have a capability of handling multi-frequency and multiconstellation GNSS. After the GEO satellite is launched, the pseudolite broadcasting system developed in phase 2 can be used in solving GEO satellite visibility problems such as with EGNOS pseudolite.

\section{Preliminary Test}

To show the initial capability of KWTB, a preliminary test was conducted via simulation. Satellite orbit and clock errors were made by RINEX navigation files and precise orbit data from IGS SP3 files. Satellite orbit and clocks calculated from SP3 are assumed as true. Ionospheric delay was generated from IONEX files. The other error sources such as tropospheric delay or receiver noise were generated by 


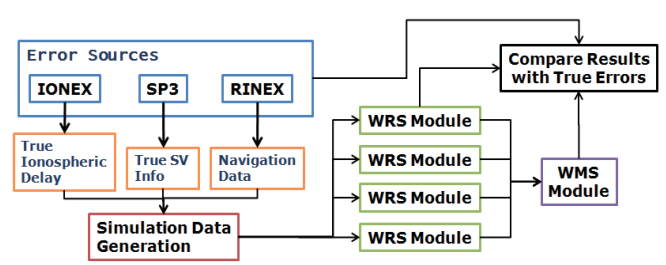

Fig. 15. Simulation scenario.

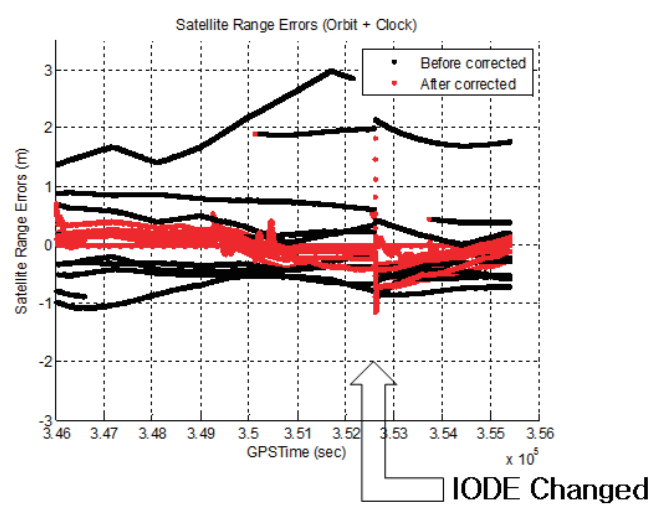

Fig. 16. Time history of satellite range errors.

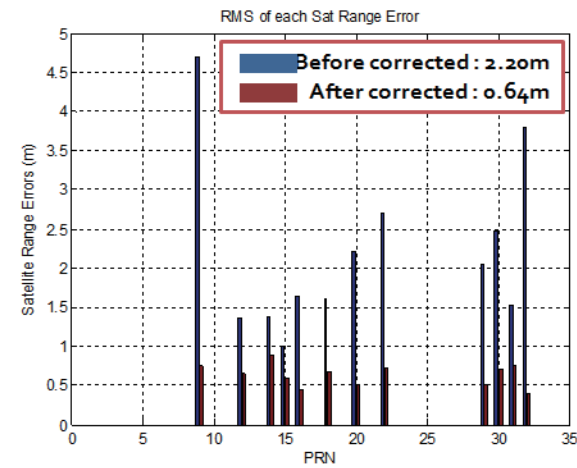

Fig. 17. Histogram of satellite range errors for each PRN.

accurate modeling. Finally, measurements were generated from these error sources. Simulation data were encoded into WRS receiver input protocol so that the WRS module takes it as the data from receiver. By comparing the results of WRS and WMS with the true data, the performance of each module can be examined numerically. Figure 15 shows a whole simulation scenario.

The simulation was conducted for 6 hours and each process was evaluated every second. In this simulation, WRSs are located at HOMI, MARA, JUMN, SRS2. The first three WRSs are in the Korean NDGPS reference stations and
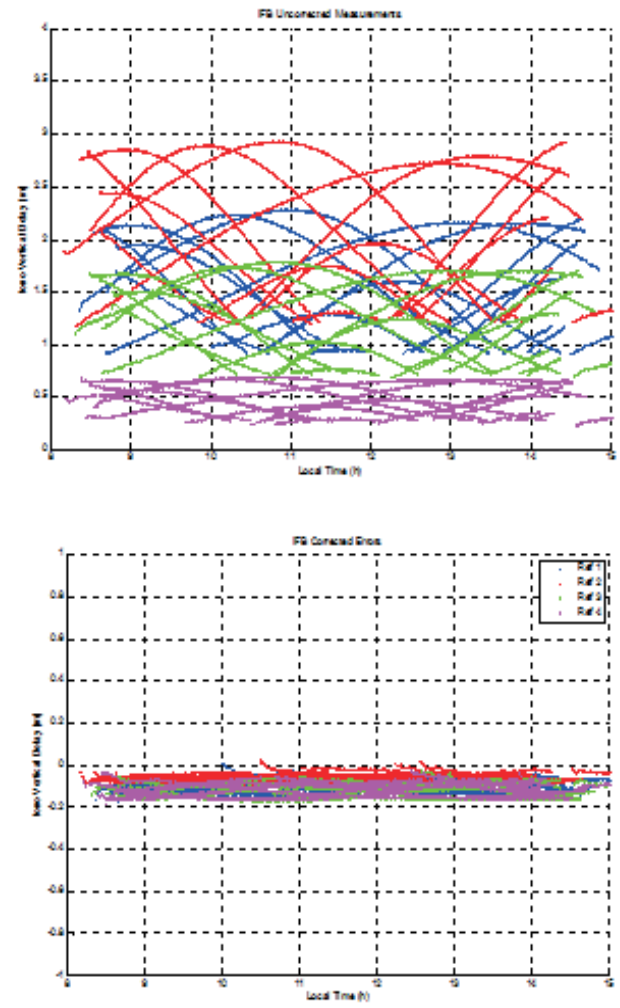

Fig. 18. Ionospheric delay estimation errors (top: Rx IFB uncorrected, bottom: Rx IFB corrected). IFB: inter-frequency bias.

the last one is located at the SNU GNSS Lab.

Figures 16 and 17 describe a satellite orbit and clock errors projected into line of sight directions. WMS estimates the satellite orbit and clock errors of the inverted GPS concept with Kalman filter. However, the estimated 3-dimensional satellite orbit errors do not follow the true satellite orbit errors exactly because network size of KWTB is very small compared to satellite geometry. Although the estimated 3-dimesional satellite orbit errors cannot follow the true orbit errors, ranging errors which affect the user position accuracy are estimated very accurately at every location in Korea. Satellite related errors are reduced to $24 \%$ after applying the KWTB satellite orbit and clock corrections.

Figure 18 shows ionospheric delay errors before and after Rx IFB compensation. When Rx IFB is not compensated for, ionospheric delays of each WRS have large errors because of Rx IFB.

Before the Rx IFB compensation, ionospheric delay estimation errors are about 1.5 meters. After the compensation, estimation errors are reduced to 0.15 meters.

Figure 19 shows true ionosphere grid point vertical delay errors (IGP VDE) around Korea, which is calculated from 

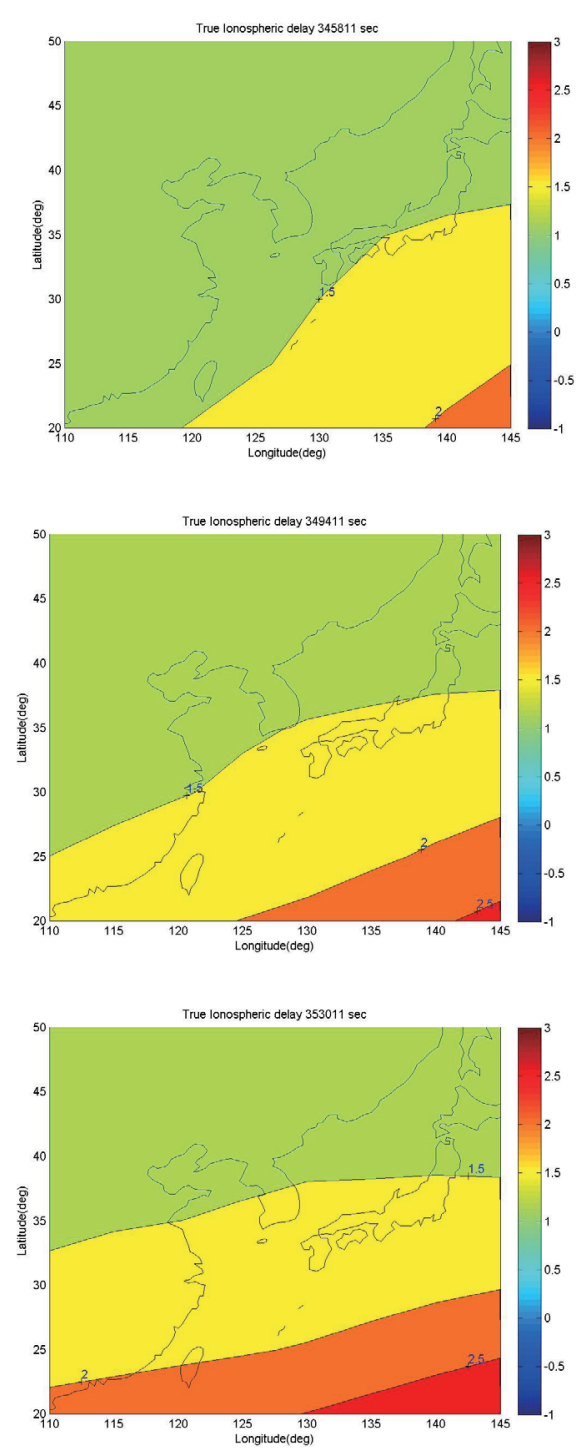

Fig. 19. True ionosphere grid point vertical delay errors (time interval: 1 hour).

IONEX data. Figure 20 shows estimated IGP VDE, which is processed by KWTB WMS. The snapshot of the ionosphere map was taken every hour. The dark blue region in Fig. 20 which has a IGP VDE value of -1 means that this IGP is not monitored in KWTB WRSs. The measurements used in the simulation are 'clean', hence ionospheric delay was estimated almost perfectly. In this simulation, ionospheric delay errors are reduced to less than $10 \%$ after applying the KWTB WMS ionospheric delay corrections.

So far, initial performance of KWTB has been evaluated by the simulation. After phase 2, the development will be completed and some estimation algorithms and integrity
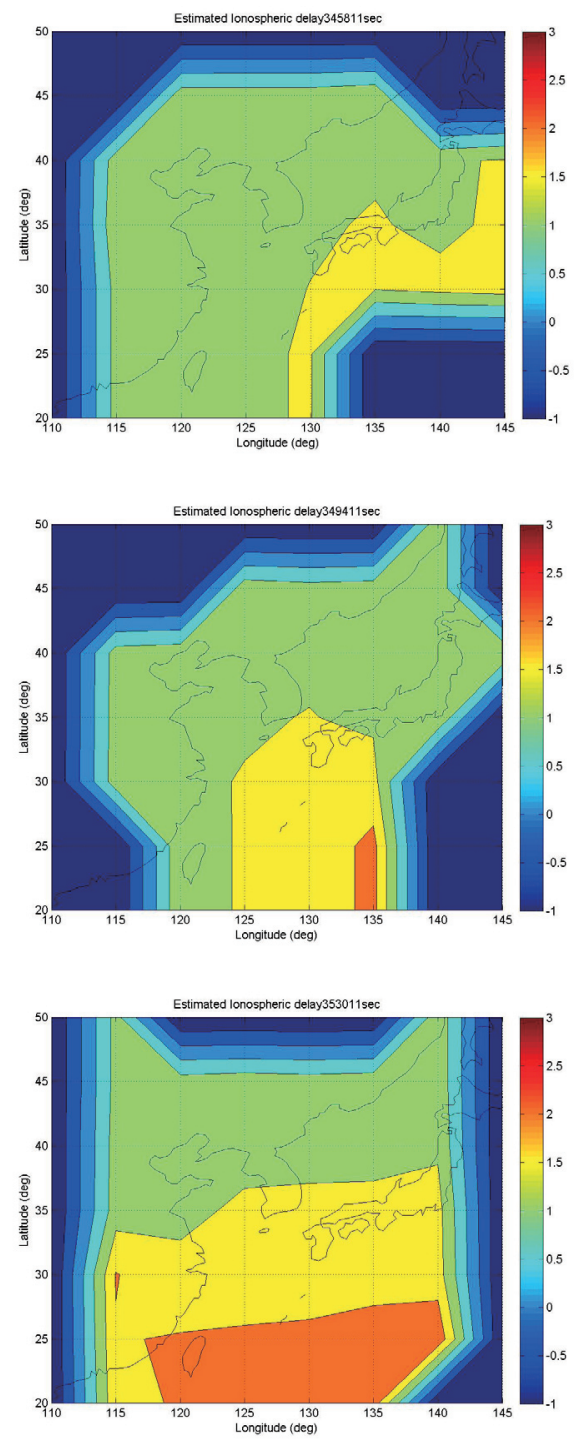

Fig. 20. Estimated ionosphere grid point vertical delay errors (time interval: 1 hour).

information generation algorithms will be improved. Therefore, the performance of the final system is expected to be improved compared with this simulation's results.

\section{Conclusions}

In this paper, the history and the current status of the Korean WADGPS development plan is presented. The Korean WADGPS development phase 1 has been successfully completed. Phase 2 has just started, with the participation of one government office and seven research institutes and 
universities. In this phase the technologies of the WADGPS ground system and pseudolite broadcasting system are secured. In phase 3, Korea will launch multi-functional GEO satellites and initial operation of Korean SBAS will be started. After this project, Korea will join the ranks of advanced countries in GNSS.

\section{Acknowledgements}

This research was supported by a grant from "Development of Wide Area DGNSS" funded by MLTM of Korean government, contracted through SNU-IAMD at SNU.

\section{References}

Chao, Y. C. (1997). Real Time Implementation of the Wide Area Augmentation System for the Global Positioning System with an Emphasis on Ionospheric Modeling. PhD Thesis,
Stanford University.

Chen, R., Hyttinen, A., Chen, Y., Strom, M., Laitinen, H., Tossaint, M., and Martin, S. (2007). Development of the EGNOS pseudolite system. Journal of Global Positioning Systems, 6, 119-125.

Kim, D. Y. and Kee, C. D. (2003). Development \& performance analysis of Korean WADGPS positioning algorithm. Wuhan University Journal of Natural Sciences, 8 , 575-580.

Radio Technical Commission for Aeronautics. (2006). Minimum Operational Performance Standards for Global Positioning System/Wide Area Augmentation System Airborne Equipment. Washington, DC: Radio Technical Commission for Aeronautics.

Tsai, Y. J. (1999). Wide Area Differential Operation of the Global Positioning System: Ephemeris and Clock Algorithms. PhD Thesis, Stanford University.

Walter, T., Hansen, A., and Enge, P. (2001). Message Type 28. Proceedings of the 2001 National Technical Meeting of The Institute of Navigation, Long Beach, CA. pp. 522-532. 